\title{
VARIAÇÃO LONGITUDINAL E EFEITO DO ESPAÇAMENTO NA MASSA ESPECÍFICA BÁSICA DA MADEIRA DE Mimosa scabrella E Ateleia glazioveana
}

\author{
Elder Eloy ${ }^{1}$, Braulio Otomar Caron ${ }^{2}$, Rômulo Trevisan ${ }^{3}$, Denise Schmidt ${ }^{2}$, Magda Lea Bolzan Zanon ${ }^{3}$, \\ Alexandre Behling ${ }^{4}$, Gean Charles Monteiro ${ }^{5}$ \\ ${ }^{1}$ Eng. Florestal, Mestrando em Agronomia, UFSM/CESNORS, Frederico Westphalen, RS, Brasil - eloyelder@yahoo.com.br \\ ${ }^{2}$ Eng. Agrônomo(a), Dr(a)., Depto. de Agronomia, UFSM/CESNORS, Frederico Westphalen, RS, Brasil - \\ otomarcaron@yahoo.com.br - denises@smail.ufsm.br \\ ${ }^{3}$ Eng. Florestal, Dr(a)., Depto. de Engenharia Florestal, UFSM/CESNORS, Frederico Westphalen, RS, Brasil - \\ romulo_trevisan@yahoo.com.br - magdazanon@smail.ufsm.br \\ ${ }^{4}$ Eng. Florestal, Mestrando em Engenharia Florestal, UFPR, Curitiba, PR, Brasil - alexandre.behling@yahoo.com.br \\ ${ }^{5}$ Eng. Agrônomo, Depto. de Agronomia, UFSM/CESNORS, Frederico Westphalen, RS, Brasil - gean.monteiro@yahoo.com.br
}

Recebido para publicação: 29/09/2012 - Aceito para publicação: 01/05/2013

\begin{abstract}
Resumo
Este trabalho teve como objetivo avaliar a variação longitudinal e o efeito do espaçamento de plantio na massa específica básica das árvores de Mimosa scabrella Benth e Ateleia glazioveana Baill, aos 36 meses de idade. Analisou-se um experimento instalado em delineamento de blocos completos casualizados, com três repetições. Os tratamentos foram definidos em função dos espaçamentos (2,0x1,0 m, 2,0x1,5 m, 3,0x1,0 m e 3,0x1,5 m). Foram avaliadas 72 árvores, sendo essas medidas e cubadas pelo método de Smalian. Em seguida, foram retirados discos nas posições do tronco: 0,10 m (base), 1,30 m do solo (DAP - Diâmetro a Altura do Peito), 25\%, 50\% e 75\% da altura total da árvore, para determinação da massa específica básica. A massa específica básica em árvores de Mimosa scabrella apresentou um decréscimo até a região do DAP, seguido de acréscimo, sem tendência de estabilização com a altura. A Ateleia glazioveana apresentou uma tendência de decréscimo no sentido base-topo das árvores. Os espaçamentos influenciaram a variação da massa específica básica em árvores de Mimosa scabrella e Ateleia glazioveana, não sendo verificada uma variação sistemática positiva ou negativa em relação ao espaço vital proporcionado.

Palavras-chave: Variação axial; característica tecnológica da madeira; qualidade da madeira; espaçamento de plantio.
\end{abstract}

\begin{abstract}
Longitudinal variation and effect of spacing on basic density wood of Mimosa scabrella and Ateleia glazioveana. This study aimed to evaluate the variation in length and effect of spacing of the basic density of Mimosa scabrella Benth e Ateleia glazioveana Baill, trees with 36 months of age. We analyzed an experiment set up in randomized complete block design with three replications. The treatments were defined by the spacing $(2.0 \times 1.0 \mathrm{~m}, 2.0 \times 1.5 \mathrm{~m}, 3.0 \times 1.0 \mathrm{~m}, 3.0 \times 1.5 \mathrm{~m}) .72$ trees were evaluated, and these measures and scaled by the method of Smalian. Then, the disks were removed from the trunk positions: $0.10 \mathrm{~m}$ (base), $1.30 \mathrm{~m}$ (DAP - diameter at breast height), $25 \%, 50 \%$ and $75 \%$ of the total height of the tree, determining the basic density. The basic density in trees of Mimosa scabrella showed a decrease to the region of the DAP, followed by addition, without a tendency to stabilize with time. The Ateleia glazioveana showed a decreasing trend towards bottom-up tree. The spacing influenced the variation of basic density in trees of Mimosa scabrella e Ateleia glazioveana not been verified a systematic positive or negative trend in relation to the living space provided. Keywords: Axial variation; technological characteristic of wood; wood quality; planting space.
\end{abstract}

\section{INTRODUÇÃO}

A madeira oriunda de florestas plantadas, por suas características próprias e pelas condições ecológicas favoráveis, tem sido apontada como uma opção lógica a ser utilizada devido a sua 
potencialidade como matéria-prima, desde que ocorra o conhecimento adequado de suas propriedades. O aprimoramento no emprego de novas tecnologias para transformação e uso racional da madeira na geração de novos produtos requer o conhecimento de suas características e variação como matéria-prima.

Para a avaliação da qualidade da madeira e definir sua melhor utilização é essencial a identificação das propriedades físicas e mecânicas que, conhecidamente, alteram o produto final. A massa específica básica desse material é considerada como o principal parâmetro tecnológico, usado para expressar a qualidade nos variados processos industriais, devido a sua íntima relação com outras propriedades e, ainda, por ser de fácil determinação (EISFELD et al., 2009; MATTOS et al., 2011). A mesma está diretamente relacionada com a estrutura anatômica e composição química, acarretando em possíveis alterações na resistência mecânica, estabilidade dimensional e qualidade da superfície usinada (LOPES et al., 2011) e, resultando em uma complexa combinação dos constituintes internos da madeira.

Conforme Melo et al. (2006) do ponto de vista da utilização tecnológica da madeira, torna-se importante o diagnóstico da variabilidade dentro da árvore, tanto no sentido radial (medula-casca) quanto no sentido longitudinal (base-topo). Essas variações da massa específica se devem às diferenças na estrutura anatômica da madeira das diversas espécies nativas ou exóticas, em menor grandeza, por quantidade de substâncias extrativas presentes por unidade de volume influenciadas, principalmente, pela idade da planta, genótipo, qualidade do material e índice de sítio, clima, condições ambientais e tratos silviculturais utilizados (TREVISAN et al., 2007).

Com relação ao sentido longitudinal, não se observa uma tendência definida de aumento ou diminuição dessa característica tecnológica. Downes e Raymond (1997) estudando a variabilidade da formação da massa específica básica da madeira de folhosas citam pelo menos quatro modelos de variação desta propriedade tecnológica: 1) Massa específica decrescendo uniformemente com a altura; 2) Massa específica decrescente até o meio do tronco e crescente deste até o topo; 3) Massa específica crescente da base para o topo, não obedecendo a um padrão uniforme e 4) Massa específica não variando conforme a altura.

Vale et al. (2009) afirmaram que durante o desenvolvimento das árvores, fatores ambientais e intrínsecos a uma própria espécie determinam o grau de variação da massa específica no sentido radial e longitudinal. Para Malan e Hoon (1992) o fator densidade populacional por meio do espaçamento e/ou desbaste, ou a combinação de ambos são métodos silviculturais que acarretam em maior desempenho na formação da planta e logo, na massa específica da madeira.

O conhecimento dos fatores que afetam a massa específica, bem como informações sobre a influência das práticas silviculturais ou sobre a relação entre a taxa de crescimento e essa característica tecnológica, ainda não são conclusivas (DEBELL et al., 2001). Para Roque e Ledzema (2003) o maior espaçamento acarretou no aumento da massa específica básica da madeira. Entretanto, Garcia et al. (1991) encontraram uma diminuição dessa característica com o aumento do espaçamento; e Vital e Della Lucia (1987), não relataram efeito significativo do espaçamento de plantio e a massa específica básica da madeira.

Partindo da hipótese de que a área útil proporcionada pelo espaçamento possa alterar as propriedades da madeira, este trabalho teve como objetivo avaliar o efeito do espaçamento na massa específica básica da madeira de Mimosa scabrella Benth e Ateleia glazioveana Baill, bem como a variação axial desta característica tecnológica em árvores de um povoamento com 36 meses de idade.

\section{MATERIAL E MÉTODOS}

O trabalho foi realizado no experimento localizado em área pertencente ao Laboratório de Agroclimatologia (LAGRO), vinculado à Universidade Federal de Santa Maria (UFSM), Campus do Centro de Educação Superior Norte do Rio Grande do Sul (CESNORS), sob coordenadas geográficas de $27^{\circ} 22^{\prime}$ 'S; $53^{\circ} 25^{\prime}$ W, a $480 \mathrm{~m}$ de altitude, no município de Frederico Westphalen - RS, aos 36 meses de idade.

Segundo a classificação climática de Köppen o clima da região é Cfa. O experimento está distante de Iraí a, aproximadamente, $30 \mathrm{~km}$, sendo o município tomado como referência para os dados de classificação climática. Conforme proposta de Maluf (2000), Iraí apresenta clima de tipo subtemperado subúmido, sendo a temperatura média anual de $18,8^{\circ} \mathrm{C}$ e temperatura média do mês mais frio de $13,3{ }^{\circ} \mathrm{C}$. 
O experimento foi instalado utilizando o delineamento experimental de blocos completos casualizados, sendo caracterizado por um fatorial 2 4 , ou seja, duas espécies florestais (Mimosa scabrella Benth e Ateleia glazioveana Baill) e quatro espaçamentos $(2,0 \times 1,0 \mathrm{~m}, 2,0 \times 1,5 \mathrm{~m}, 3,0 \times 1,0 \mathrm{~m}$ e 3,0 x $1,5 \mathrm{~m}$ ) em três repetições. O bloco contempla 16 unidades experimentais, sendo que cada uma possui 45 plantas distribuídas em cinco linhas.

Foram abatidas 72 árvores, sendo 36 para cada espécie, em que foram retirados discos com, aproximadamente, dois centímetros de espessura, nas seguintes posições no tronco: $0,10 \mathrm{~m}$ (base), 1,30 m do solo (DAP), 25\%, 50\% e 75\% da altura total da árvore. Os discos foram numerados de acordo com sua posição relativa no tronco e localização experimental. Em seguida, os mesmos foram acondicionados em sacos plásticos e transportados para o Laboratório de Agroclimatologia UFSM / CESNORS, onde foram marcadas e seccionadas duas cunhas simetricamente opostas.

As cunhas de madeira foram submersas em água, onde permaneceram até atingirem peso constante. Para a obtenção da massa específica básica de cada cunha $\left(\rho_{\mathrm{b}}\right), \mathrm{em} \mathrm{g} / \mathrm{cm}^{3}$, determinou-se, o volume verde $(\mathrm{Vu}), \mathrm{em} \mathrm{cm}^{3}$, utilizando o método da balança hidrostática, descrito por Vital (1984), e a massa seca (Mo), em g, que foi obtida deixando-se as amostras em estufa a $103{ }^{\circ} \mathrm{C}$ até peso constante, através do emprego da seguinte expressão:

$$
\rho_{\mathrm{b}=} \mathrm{Mo} / \mathrm{Vu} .
$$

A massa específica básica da madeira em cada posição relativa do tronco foi determinada pela média dos valores das duas cunhas. Em seguida, calculou-se a massa específica básica ponderada $\left(\rho_{\mathrm{b} \text { pond. }}\right)$ em função do volume total sem casca de cada árvore, $\mathrm{em} \mathrm{g} / \mathrm{cm}^{3}$. Esse cálculo baseia-se na ponderação dos valores de massa específica básica obtida em cada posição relativa $\left(\rho_{\mathrm{b}}{ }^{\prime} \mathrm{i}^{\prime}\right)$, em $\mathrm{g} \mathrm{cm}^{-3}$; os volumes sem casca correspondentes entre duas posições sucessivas $\left(\mathrm{v}_{1}, \mathrm{v}_{2}, \mathrm{v}_{\mathrm{i}}\right), \mathrm{em} \mathrm{m}^{3}$; com o volume total sem casca da árvore $\left(\mathrm{v}_{\mathrm{s}}\right)$, em $\mathrm{m}^{3}$, definido pelo método de Smalian (FINGER, 1992). Assim, a massa específica básica ponderada foi definida pela seguinte expressão:

$$
\rho_{\mathrm{b} \text { pond }}=\left(\left(\left(\left(\rho_{\mathrm{b} 0 \%}+\rho_{\mathrm{bDAP}}\right) / 2\right) * \mathrm{v}_{1}\right)+\left(\left(\left(\rho_{\mathrm{bDAP}}+\rho_{\mathrm{b} 25 \%}\right) / 2\right) * \mathrm{v}_{2}\right)+\left(\left(\left(\rho_{\mathrm{bi}}+\rho_{\mathrm{bi}+1}\right) / 2\right) * \mathrm{v}_{\mathrm{i}}\right)\right) / \mathrm{v}_{\mathrm{s}} .
$$

Para a análise do efeito do espaçamento na massa específica básica das árvores, os dados amostrados por posição relativa foram submetidos à análise de regressão. Estes foram processados pelo software "Statistical Analysis System" (SAS, 1993), no qual foi, primeiramente, aplicado o procedimento de seleção de variáveis Stepwise. O modelo foi definido pela massa específica básica das árvores $\left(\rho_{\mathrm{b}}\right)$, em $\mathrm{g} / \mathrm{cm}^{3}$, em função das posições relativas no sentido base-topo $(\mathrm{P})$, em \%, pela da equação:

$$
\rho_{\mathrm{b}}=\mathrm{f}\left(\mathrm{P} ; 1 / \mathrm{P} ; \mathrm{P}^{2} ; 1 / \mathrm{P}^{2} ; \ln \mathrm{P} ; 1 / \ln \mathrm{P} ; \sqrt{ } \mathrm{P}\right) .
$$

O melhor modelo foi selecionado quanto as condicionantes da regressão com base no coeficiente de determinação ajustado $\left(\mathrm{R}_{\text {aj }}^{2}\right)$, coeficiente de variação $(\mathrm{CV})$, erro padrão da estimativa $\left(\mathrm{S}_{\mathrm{xy}}\right)$, e análise da distribuição dos resíduos.

Após esse procedimento, no modelo escolhido para descrever a variação axial da massa específica foram adicionadas variáveis Dummy, que assumiram valores de 0 e 1 , conforme o espaçamento de plantio aplicado, da seguinte forma: $\mathrm{Di}=1$, se a árvore estivesse presente no espaçamento $\mathrm{i}$; e $\mathrm{Di}=0$, se a árvore estivesse ausente nesse espaçamento $\mathrm{i}$.

Com esse método foi possível expressar as regressões individuais ajustadas para os quatro espaçamentos estudados em função de uma regressão linear múltipla, representada pelas variáveis independentes descritas na seguinte equação:

$$
\rho_{\mathrm{b}=\mathrm{f}}(\mathrm{X} ; \mathrm{Di} ; \mathrm{Di} . \mathrm{X}) \text {. }
$$

Em que $\rho_{b}=$ massa específica básica das árvores, em $\mathrm{g} / \mathrm{cm}^{3} ; \mathrm{X}=$ posição relativa no sentido base-topo selecionada pelo procedimento Stepwise de regressão, em \%; Di = Dummy (espaçamento de plantio, onde $\mathrm{i}=1 ; \ldots ; 4) ;$ Di. $X=$ interação variável Di com a variável $\mathrm{X}$. 


\section{RESULTADOS E DISCUSSÃO}

Os valores médios da massa específica básica por posição no sentido base-topo e da massa específica básica ponderada em função do volume total sem casca, assim como a altura total e o diâmetro a altura do peito das espécies florestais Mimosa scabrella e Ateleia glazioveana distribuídas em diferentes espaçamentos de plantio, aos três anos de povoamento, são destacados na tabela 1.

Tabela 1. Massa específica básica média por posição no sentido axial, massa específica básica ponderada em função do volume total sem casca, altura total e o diâmetro a altura do peito (DAP) para as árvores de Mimosa scabrella e Ateleia glazioveana em diferentes espaçamentos de plantio.

Table 1. Basic density per position in the axial direction, basic density-weighted total shelled, total height and diameter at breast height (DAP) trees Mimosa scabrella and Ateleia glazioveana in different planting spacings.

\begin{tabular}{|c|c|c|c|c|c|c|c|c|c|}
\hline \multirow{2}{*}{ Espécie } & \multirow{2}{*}{$\begin{array}{c}\text { Espaçamento } \\
\text { (m) }\end{array}$} & \multirow{2}{*}{$\begin{array}{c}\text { Altura } \\
\text { (m) }\end{array}$} & \multirow{2}{*}{$\begin{array}{l}\text { DAP } \\
\text { (cm) }\end{array}$} & \multicolumn{5}{|c|}{ Posição no sentido base-topo } & \multirow{2}{*}{$\rho_{\text {pond. }}(\sigma)$} \\
\hline & & & & $0,1 \mathrm{~m}$ & DAP & $25 \%$ & $\mathbf{5 0 \%}$ & $75 \%$ & \\
\hline \multirow[t]{4}{*}{ Mimosa scabrella } & $2,0 \times 1,0$ & 7,39 & 18,28 & 0,419 & 0,393 & 0,399 & 0,436 & 0,416 & $0,406( \pm 0,015)$ \\
\hline & $2,0 \times 1,5$ & 7,59 & 20,08 & 0,417 & 0,378 & 0,399 & 0,391 & 0,373 & $0,400( \pm 0,016)$ \\
\hline & $3,0 \times 1,0$ & 5,40 & 14,76 & 0,448 & 0,389 & 0,39 & 0,408 & 0,432 & $0,407( \pm 0,023)$ \\
\hline & $3,0 \times 1,5$ & 5,54 & 16,80 & 0,432 & 0,386 & 0,399 & 0,424 & 0,429 & $0,414( \pm 0,018)$ \\
\hline \multirow{4}{*}{$\begin{array}{l}\text { Ateleia } \\
\text { glazioveana }\end{array}$} & $2,0 \times 1$ & 5,00 & 17,79 & 0,477 & 0,474 & 0,483 & 0,462 & 0,439 & $0,473( \pm 0,016)$ \\
\hline & $2,0 \times 1,5$ & 6,81 & 18,13 & 0,479 & 0,475 & 0,468 & 0,45 & 0,442 & $0,468( \pm 0,014)$ \\
\hline & $3,0 \times 1,0$ & 4,29 & 17,17 & 0,493 & 0,488 & 0,482 & 0,48 & 0,455 & $0,489( \pm 0,013)$ \\
\hline & $3,0 \times 1,5$ & 4,36 & 17,89 & 0,503 & 0,486 & 0,472 & 0,472 & 0,45 & $0,486( \pm 0,018)$ \\
\hline
\end{tabular}

$\rho_{\text {pond.: }}$ massa específica básica ponderada em função do volume total sem casca da árvore, $\mathrm{g} / \mathrm{cm}^{3}$; DAP: diâmetro a altura do peito; $\sigma$ : desvio padrão, $\mathrm{g} / \mathrm{cm}^{3}$.

A massa específica básica encontrada para as árvores de Mimosa scabrella e Ateleia glazioveana, em todos os tratamentos, foram menores do que as citadas na literatura para as mesmas espécies. Para a Mimosa scabrella em árvores com 8 anos de idade, Sturion e Tomaselli (1990) observaram valores para esta característica tecnológica de $0,521 \mathrm{~g} / \mathrm{cm}^{3}$. Já Jankowsky et al. (1990) relataram valores que variaram entre 0,510 a $0,610 \mathrm{~g} / \mathrm{cm}^{3}$, confirmando a existência de variabilidade dentro da espécie.

Para a Ateleia glazioveana, os valores de massa específica básica encontrados na literatura variaram de 0,500 a $0,760 \mathrm{~g} / \mathrm{cm}^{3}$ (CARVALHO, 2002). Essa diferença entre valores de massa específica, segundo Silva et al. (2004) e Alzate et al. (2005), pode ser atribuída à idade e, principalmente, a fatores ambientais e/ou genéticos.

A equação de regressão selecionada para a espécie Mimosa scabrella $\left(\rho_{b}=0,39335+0,04264(1 / P)+0,00000357\left(P^{2}\right)\right)$ para estimar a variação axial da massa específica básica nas árvores $\left(\rho_{\mathrm{b}}\right)$, em relação à posição relativa no sentido base-topo $(\mathrm{P})$, apresentou um coeficiente de variação igual a $9,2 \%$, coeficiente de determinação ajustado de 0,06 e erro padrão da estimativa de 0,037 $\mathrm{g} / \mathrm{cm}^{3}$.

Para a espécie Ateleia glazioveana a equação de regressão selecionada ( $\rho_{b}=0,49413$ $0,00059486(\mathrm{P}))$ para estimar a variação axial da massa específica básica nas árvores $\left(\rho_{\mathrm{b}}\right)$, em relação à posição relativa no sentido base-topo $(\mathrm{P})$, apresentou um coeficiente de variação igual a $6,8 \%$, coeficiente de determinação ajustado de 0,17 e erro padrão da estimativa de $0,032 \mathrm{~g} / \mathrm{cm}^{3}$.

Com base nos modelos selecionados, os dados de massa específica básica em função da posição relativa no sentido base-topo foram então relacionados com os respectivos espaçamentos de plantio e submetidos à análise de regressão com o emprego de variável Dummy (Tabela 2).

As equações selecionadas para Mimosa scabrella e Ateleia glazioveana (Tabela 2) não podem ser utilizadas para todos os espaçamentos, pois a Dummy D2, empregada para verificar a influência do espaçamento $2(2,0 \times 1,5 \mathrm{~m})$, na massa específica básica, foi significativa $(\mathrm{p}<0,05)$ para as duas espécies. $\mathrm{Na}$ mesma tabela, verifica-se, ainda, que não houve interação significativa entre os espaçamentos e a posição relativa no sentido base-topo. 
Tabela 2. Análise de variância da regressão reajustada com variável Dummy (tipo SS1) da massa específica básica da madeira de Mimosa scabrella e Ateleia glazioveana em função da posição relativa e do espaçamento.

Table 2. Analysis of variance adjusted regression with dummy variable (type SS1) of the basic density of wood Mimosa scabrella and Ateleia glazioveana depending on the relative position and spacing.

\begin{tabular}{|c|c|c|c|c|c|}
\hline \multicolumn{3}{|c|}{ Mimosa scabrella } & \multicolumn{3}{|c|}{ Ateleia glazioveana } \\
\hline FV & GL & QM & FV & GL & QM \\
\hline Modelo & 9 & $0,00450143 *$ & Modelo & 7 & $0,00630768^{*}$ \\
\hline $1 / \mathrm{P}$ & 1 & $0,01061117^{*}$ & $\mathrm{P}$ & 1 & $0,03569771^{*}$ \\
\hline $\mathrm{P}^{2}$ & 1 & $0,00698124 *$ & - & - & - \\
\hline D1 & 1 & $0,00022507^{\mathrm{ns}}$ & D1 & 1 & $0,00019447^{\text {ns }}$ \\
\hline D2 & 1 & $0,00874115^{*}$ & D2 & 1 & $0,00735189 *$ \\
\hline D3 & 1 & $0,00007344^{\mathrm{ns}}$ & D3 & 1 & $0,00020939^{\text {ns }}$ \\
\hline D4 & 0 & 0 & D4 & 0 & - \\
\hline $\mathrm{D} 1.1 / \mathrm{P}$ & 1 & $0,00384078^{\mathrm{ns}}$ & D1.P & 1 & $0,00044409^{\text {ns }}$ \\
\hline $\mathrm{D} 2.1 / \mathrm{P}$ & 1 & $0,00375306^{\mathrm{ns}}$ & D2.P & 1 & $0,00000840^{\mathrm{ns}}$ \\
\hline D3.1/P & 1 & $0,00178553^{\text {ns }}$ & D3.P & 1 & $0,00024780^{\text {ns }}$ \\
\hline D4.1/P & 0 & 0 & D4.P & 0 & - \\
\hline Erro & 153 & 0,00132466 & Erro & 160 & 0,00102068 \\
\hline Total & 162 & - & Total & 167 & - \\
\hline
\end{tabular}

Assim, os valores de massa específica da madeira de Mimosa scabrella foram estimados para os espaçamentos por meio da equação: $\rho b=0,397258+0,044746(1 / P)+0,00000364\left(\mathrm{P}^{2}\right)-0,016575 \mathrm{D} 2$, em que: $\rho \mathrm{b}=$ massa específica básica, $\mathrm{g} / \mathrm{cm}^{3} ; \mathrm{P}=$ posição relativa no sentido base-topo, $\% ; \mathrm{D} 2=$ variável Dummy correspondente ao espaçamento $2(2,0 \times 1,5 \mathrm{~m})$, com coeficiente de determinação ajustado de 0,15 , coeficiente de variação igual a $8,9 \%$ e valor de erro padrão da estimativa de $0,036 \mathrm{~g} / \mathrm{cm}^{3}$. Já para a Ateleia glazioveana a equação de regressão que estimou os valores de massa específica foi dada por: $\rho b=0,497933-0,000602(\mathrm{P})-0,013496 \mathrm{D} 2$, apresentando coeficiente de determinação ajustado de 0,20, coeficiente de variação igual a $6,7 \%$ e valor de erro padrão da estimativa de $0,031 \mathrm{~g} / \mathrm{cm}^{3}$.

Os valores de massa específica básica da madeira de Mimosa scabrella e Ateleia glazioveana, em função da posição relativa no sentido base-topo, estimados para os quatro espaçamentos de plantio através dos modelos anteriormente descritos, podem ser observados na figura $1 \mathrm{~A} \mathrm{e} \mathrm{1B}$, respectivamente.

Para a espécie Mimosa scabrella os valores dessa característica tecnológica decrescem da base até, aproximadamente, a região do DAP. A partir dessa posição, os valores são crescentes, sem tendência de estabilização com a altura (Figura 1A). Os resultados encontrados corroboram com os descritos por Malan e Hoon (1992); Trevisan et al. (2012) para a espécie Eucalyptus grandis, que constataram a diminuição da massa específica até a região do DAP, seguida de aumento a partir dessa posição. Em contrapartida, para Ateleia glazioveana observou-se uma tendência de decréscimo da massa específica básica da base até a região que corresponde ao topo das árvores (Figura 1B).

Observou-se que houve influência das diferentes formas de competição proporcionadas pelos espaçamentos (Figura 1), porém sem uma tendência sistemática positiva ou negativa definida em relação ao espaço vital, mas sim com uma interação entre essas sistemáticas. Dessa forma, independentemente do espaçamento aplicado, não existe um padrão na variação da massa específica básica da madeira em função da posição relativa no sentido base-topo.

Estes resultados são divergentes dos descritos por Vital e Della Lucia (1987), que não encontraram efeito significativo do espaçamento na massa específica da madeira. Já Roque e Ledzema (2003), verificaram um aumento da massa específica com o aumento do espaçamento. Em contrapartida, Garcia et al. (1991) encontraram uma diminuição da massa específica da madeira com o aumento do 
espaçamento. Para Trevisan et al. (2007) essas divergências de resultados podem ser decorrentes de diversos fatores, como a variabilidade genética dos povoamentos originados de sementes.
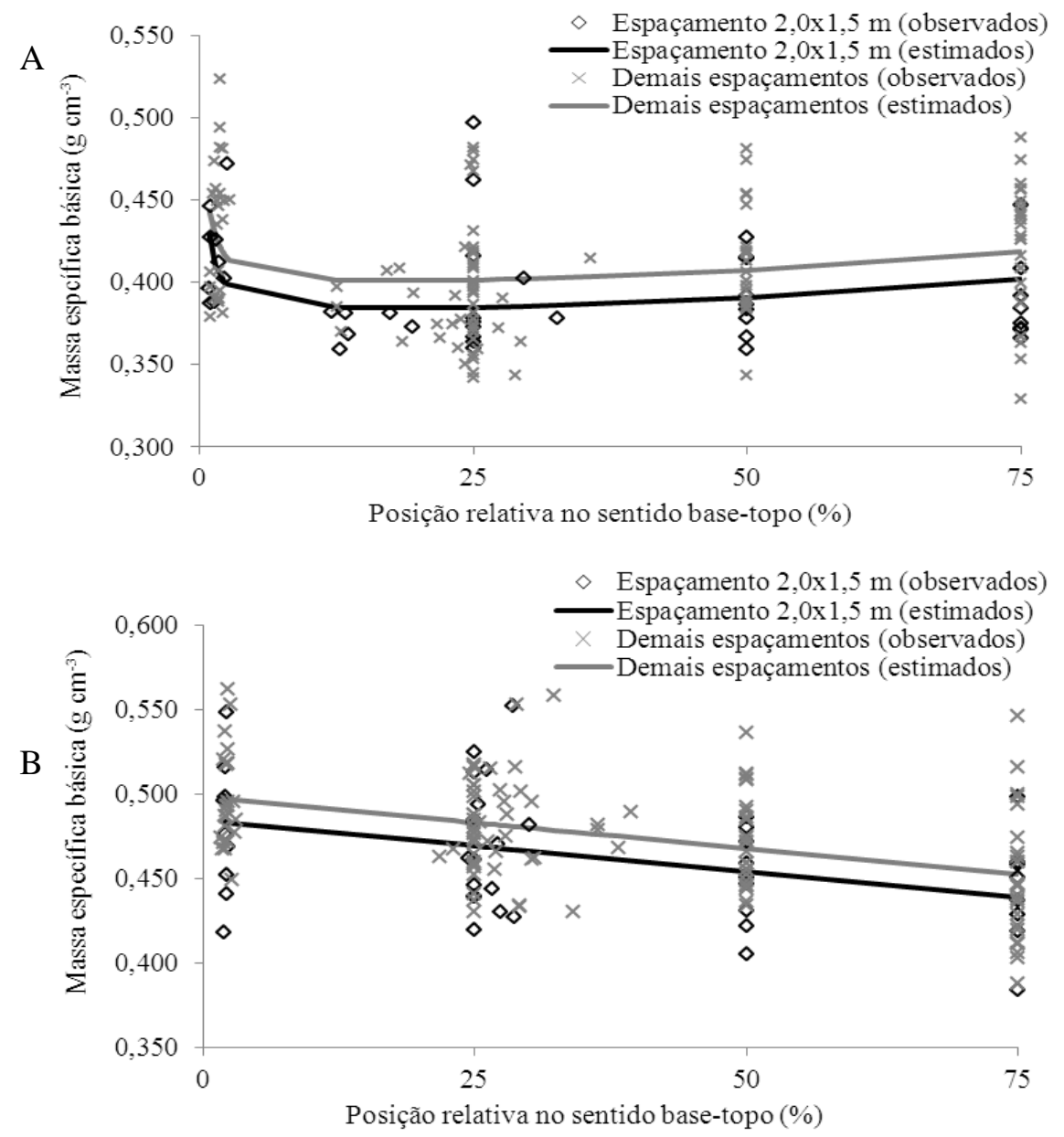

Figura 1. Variação da massa específica básica das árvores de Mimosa scabrella (A) e Ateleia glazioveana (B) em função da posição relativa no sentido axial.

Figure 1. Variation of basic density trees Mimosa scabrella (A) and Ateleia glazioveana (B) as a function of relative position in the axial direction.

\section{CONCLUSÕES}

- O padrão de variação da massa específica básica no sentido longitudinal, aos 36 meses de idade, em árvores de Mimosa scabrella, apresentou um decréscimo da base até a região do DAP, seguido de acréscimo, sem tendência de estabilização com a altura.

- Para a espécie Ateleia glazioveana observou-se uma tendência de decréscimo desta característica tecnológica da base até o topo das árvores.

- Os diferentes espaçamentos influenciaram a variação da massa específica básica em árvores de Mimosa scabrella e Ateleia glazioveana, não sendo verificada uma variação sistemática positiva ou negativa em relação ao espaço vital proporcionado pelo espaçamento.

\section{AGRADECIMENTOS}

Ao CNPq pelo apoio financeiro. 


\section{REFERÊNCIAS}

ALZATE, S. B. A.; TOMAZELO FILHO, M.; PIEDADE, S. M. S. Variação longitudinal da densidade básica da madeira de clones de Eucalyptus grandis Hill ex Maiden, E. saligna Sm. e E. grandis x urophylla. Scientia Forestalis, Piracicaba, n. 68, p. 87 - 95, 2005.

CARVALHO, P. E. Taxonomia do Timbó. Circular Técnica 57. Colombo-PR, 2002.

DEBELL, D. S.; KEYES, C. R.; GARTNER, B. L. Wood density of Eucalyptus saligna grown in Hawaiian plantations: effects of silvicultural practices and relation to growth rate. Australian Forestry, v. 64 , n. 2, p. $106-110,2001$.

DOWNES, G. M.; RAYMOND, C. A. Variation in wood density in plantation eucalyptus. In: DOWNES, G. M.; HUDSON, I.; RAYMOND, C. A.; DEAN, G. H.; MICHELL, A. J.; SCHIMLECK, L. R. Sampling plantation Eucalypts for wood and fibre properties. Australia: CSIRO Publishing, 1997. p. $88-99$.

EISFELD, R.; EISFELD, C. L.; FERREIRA, J. M.; PIMENTEL, F. R.; ALMEIDA, A. Modelagem da massa específica básica dos cavacos a partir de variáveis dendrométricas e da massa específica do disco. Floresta, Curitiba, v. 39, n. 4, p. 877 - 886, 2009.

FINGER, C. A. G. Fundamentos de Biometria Florestal. Santa Maria: UFSM/ CEPEF/ FATEC, 1992. $269 \mathrm{p}$.

GARCIA, C. H.; CORRADINI, L.; ALVARENGA, S. F. Comportamento florestal do Eucalyptus grandis e Eucalyptus saligna em diferentes espaçamentos. IPEF, p. 1 - 8, 1991. (Circular Técnica, 179).

JANKOWSKY, I. P.; CHIMELO, J. P.; CAVALCANTE, A. A.; GALINA, I. C. M.; NAGAMURA, J. C. S. Madeiras brasileiras. Caxias do Sul: Spectrum, 1990. 172 p.

LOPES, C. S. D.; NOLASCO, A. M.; TOMAZELLO FILHO, M.; DIAS, C. T. S.; PANSINI, A. Estudo da massa específica básica e da variação dimensional da madeira de três espécies de eucalipto para a indústria moveleira. Ciência Florestal, Santa Maria, v. 21, n. 2, p. 315 - 322, 2011.

MALAN, F. S.; HOON, M. Effect of initial spacing and thinning on some wood properties of Eucalyptus grandis. South African Forestry Journal, Pretoria, n. 163, p. 13 - 20, 1992.

MALUF, J. R. T. Nova classificação climática do Estado do Rio Grande do Sul. Revista Brasileira de Agrometeorologia, Santa Maria, v. 8, n. 1, p. 141 - 150, 2000.

MATTOS, B. D.; GATTO, D. A.; STANGERLIN, D. M.; CALEGARI, L.; MELO, R. R.; SANTINI, E. J. Variação axial da densidade básica da madeira de três espécies de gimnospermas. Revista Brasileira de Ciências Agrárias, Recife, v. 6, n. 1, p. 121 - 126, 2011.

MELO, R. R.; PAES, J. B.; LIMA, C. R.; FERREIRA, A. G. Estudo da Variação da Densidade Básica de Sete Madeiras do Semi-Árido. Revista Cientifica Eletrônica de Engenharia Florestal, Garça, v. 4, n. 7, 2006.

ROQUE, R. M.; LEDZEMA, R. M. Efecto del espaciamiento en plantación sobre dos propiedades físicas de madera de teca a lo largo del fuste. Madera y Bosques, Heredia, v. 9, n. 2, p. 15 - 27, 2003.

STATISTICAL ANALYSIS SYSTEM - SAS: Programa de computador, ambiente VM. Cary, 1993. Versão 6.08.

SILVA, J. C.; OLIVEIRA, J. T. S.; TOMAZELlO FILHO, M.; JÚNIOR, S. K.; MATOS, J. L. M. Influência da idade e da posição radial na massa específica básica da madeira de Eucalyptus grandis Hill ex Maiden. Revista Floresta, Curitiba, v. 34, n. 1, p. 13 - 22, 2004.

STURION, J. A.; TOMASELLI, I. Influência do tempo de estocagem de lenha de bracatinga na produção de energia. Boletim de Pesquisa Florestal, Colombo, n. 21, p. 37 - 47, 1990. 
TREVISAN, R.; HASELEIN, C. R.; SANTINI. E. J.; SCHINEIDER. P. R.; MENEZES. L. F. Efeito da intensidade de desbaste nas características dendrométricas e tecnológicas da madeira de Eucalyptus grandis. Ciência Florestal, Santa Maria, v. 17, n. 4, p. 377 - 387, 2007.

TREVISAN, R.; ELOY, E.; DENARDI, L.; HASELEIN, C. R.; CARON.B. O. Variação axial e efeito do desbaste na massa específica das árvores centrais de Eucalyptus grandis. Ciência Rural, Santa Maria, v. 42, n. 2, p. $312-318,2012$.

VALE, A. T.; ROCHA, L. R.; MENEZZI, C. H. S. D. Massa específica básica da madeira de Pinus caribaea var. hondurensis cultivado em cerrado. Scientia Forestalis, v. 37, n. 84, p. 387 - 394, 2009.

VITAL, B. R. Métodos de determinação da densidade da madeira. Viçosa: SIF/UFV. p. 1 - 21, 1984. (Boletim Técnico, 1).

VITAL, B. R.; DELLA LUCIA, R. M. Efeito do espaçamento na produção em peso e na qualidade da madeira de Eucalyptus grandis e Eucalyptus saligna aos 52 meses de idade. Revista Árvore, v. 11, n. 2, p. $132-145,1987$. 